\title{
The Influence of Emotional State on Mobile Phone Addiction Tendency in College Students: The Mediation Role of Regulatory Emotion Self-efficacy
}

\author{
Guifang $\mathrm{Fu}^{1}$, Jingwen $\mathrm{Liu}^{1}$, Haichun Liu ${ }^{2}$ \\ ${ }^{1}$ Department of Applied Psychology, School of Public Administration, Guangdong University of Foreign Studies, \\ Canton, China \\ ${ }^{2}$ School of Innovation and Entrepreneurship Education, Guangdong University of Foreign Studies, Canton, China \\ Correspondence: Liu Haichun, School of Innovation and Entrepreneurship Education, Guangdong University of \\ Foreign Studies, Canton, China.
}

Received: Feb. 3, 2020

Accepted: Mar. 9, 2020

Online Published: Mar. 13, 2020

doi:10.11114/ijce.v3i1.4764

URL: https://doi.org/10.11114/ijce.v3i1.4764

\begin{abstract}
In order to explore the situation of college students' emotional state, regulatory emotion self-efficacy and mobile phone addiction tendency as well as their relationships, a total of 350 college students were assessed with Mobile Phone Addiction Tendency Scale (MPATS), Positive Affect and Negative Affect Scale (PANAS) and the Scale of Regulatory Emotional Self-efficacy (SRESE). The result showed that: (1) $40.86 \%$ of college students had the tendency of cell phone addiction, which was serious; $72.0 \%$ of college students were in a positive emotional state, $22 \%$ were in a negative emotional state; college students' regulatory emotion self-efficacy was in the middle level; (2) there was no gender, grade, major type, household registration type (rural and urban) and whether only child difference in mobile phone addiction tendency; (3) the positive emotions of college students were negatively correlated with the tendency of mobile phone addiction, while the negative emotions were positively correlated with the tendency of mobile phone addiction, and the positive emotion was positively correlated with regulatory emotional self-efficacy; (4) the regulatory emotion self-efficacy had a partial mediating effect between the positive emotions and mobile phone addiction tendency and had no mediating effect between the negative emotions and mobile phone addiction tendency.
\end{abstract}

Keywords: emotional state, regulatory emotion self-efficacy, mobile phone addiction tendency, college student

\section{Introduction}

In 2018 the Statistical Bulletin of the Communications Operations Industry of China data showed the mobile phone user penetration rate had reached 112.2 units / 100 people and college students were the mainstream population for mobile phone use. While enjoying all kinds of new functions of mobile phones, the problem of mobile phone overuse has attracted more and more attention. Studies have shown that mobile phone overuse can bring about physiological and psychological effects. For a long or frequent use of mobile phones, individuals' sensitivity to vision and hearing will decrease; numbness, pain and other symptoms may occur in the arms, joints, cervical spine and other body parts, which will affect the quality of sleep and exercise quality of individuals (Lepp et al., 2015). Overuse of mobile phones can lead to more negative emotions, such as a strong sense of loneliness (Zhang et al., 2015) , depression and anxiety, and then had an impact on individual social performance, such as interpersonal relationship and academic achievement (Elhai et al., 2019). Therefore, it is urgent to explore the influencing factors of mobile phone addiction tendency and make reasonable suggestions and interventions.

People's research on "addiction" has gone through American psychologist Goldberg's substance addiction, Griffith's behavioral addiction, and then technology addiction in the information age. Young thought that technology addiction was a state of excessive psychological dependence of users on a certain technology, and mobile phone addiction was a kind of technology addiction (Chai, 2017). At present, there were different opinions on the research of mobile phone addiction. For example, mobile phone dependence is considered as problematic cell phone use (Bianchi, 2005); the habitual use of mobile phones for a long time that people can not leave the mobile phones, and eventually develop into various adverse consequences, which can cause harm to individuals and Society (Park, 2005); the behavior of mobile 
phone that is out of control physically and mentally and causes unhealthy (Zhang et al., 2018). In terms of influencing factors of mobile phone overuse, some scholars have studied the relationship between mobile phone addiction and loneliness, motivation of mobile phone use of college students (Liu Hong \&Wang Hongli, 2011), emotion, personality traits and social support. In this paper, mobile phone addiction tendency is defined as the negative influence of physical and mental health and social environment caused by inappropriate use of mobile phones. Mobile phone addiction tendency is the same as mobile phone dependence.

Emotional state can be divided into positive emotions and negative emotions. Negative emotions are feelings of low subjective feelings, such as anger, shame, tension, fear and so on. A study showed that the use of mobile phones in high school was related to depression, tension and excitement. Because tension and excitement is a subcomponent of anxiety, anxiety may be a potential factor in mobile phone dependence (Ikeda \& Nakamura, 2014). In addition, Coyne etc. suggested problematic cell phone use was correlated with lower well-being, lower life satisfaction and mindfulness (Coyne et al., 2019).

There were few studies on emotional state and addiction tendency of mobile phone. Therefore, this study puts forward hypothesis 1: Positive emotion was significant negative correlated with college students' mobile phone addiction tendency; hypothesis 2: Negative emotion was significant positive correlated with college students' mobile phone addiction tendency.

Bandura, an American psychologist, first put self-Efficacy forward. Since then, it has been studied extensively and continuously enriched and improved. Self-efficacy refers to a person's ability to judge, believe in, or perceives himself or herself to accomplish a certain behavior at a certain level (Bandura, 1986). Caprara put self-efficacy of emotion regulation forward. He found that the difference in management level of individual emotion could lead to different emotional experience of individuals (Caprara, 1999). After further research, they divided the self-efficacy of emotion regulation into three dimensions: the ability to identify emotional states, the ability to understand others' feelings, and the ability to manage positive and negative emotional expressions. Thus, self-efficacy of emotion regulation is whether an individual has self-confidence to regulate his emotional state (Bandura \& Caprara, 2003). Researches showed that self-efficacy of emotion regulation can play a positive role in coping with stress and interpersonal problems, thereby improving the subjective well-being of individuals, and also play an important role in regulating prosocial behavior, criminal behavior, depression, addiction behavior (Tang et al., 2010; Wen et al., 2009). At present, there are few researches on the relationship between self-efficacy of emotion regulation and mobile phone addiction. Therefore, this study aims to study self-efficacy of emotion regulation as an intermediary variable to the relationship between emotional state and mobile phone addiction tendency, and hypothesis 3 is as follows: self-efficacy of emotion regulation plays an intermediary role between emotional state (positive emotions and negative emotions) and college students' mobile phone addiction tendency.

This study aims to explore the current situation and relationship between college students' emotional state and mobile phone addiction tendency as well as the mediating effects of regulatory emotion self-efficacy or not. The theoretical mediation model is shown in Figure 1.

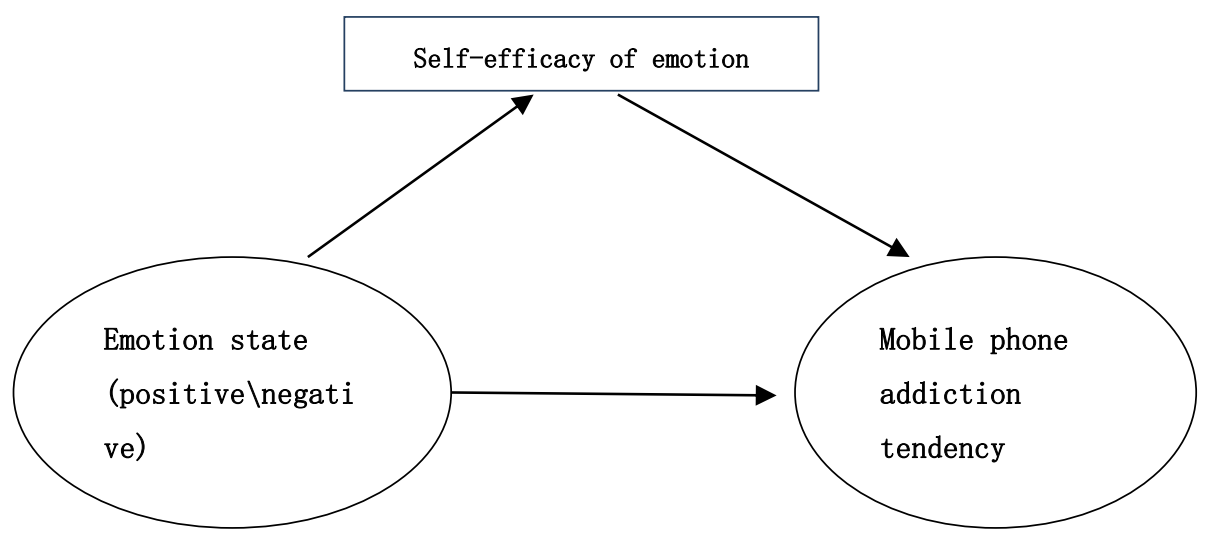

Figure 1. Intermediate Model of regulatory emotion self-efficacy

\section{Method}

\subsection{Participants}

378 college students were tested online and 350 valid questionnaires were collected (92.60\%). Among them there are 113 males and 237 females; 57 majors in literature and history, 132 in science and technology, 109 in management and 
52 in other fields; 192 rural and 158 urban; 106 in only children and 244 in non-only children.

\subsection{Measures}

\subsubsection{Mobile Phone Addiction Tendency Scale}

The 16-items Mobile Phone Addition Tendency Scale ((MPATS, Xiong et al. , 2012) was used to measured mobile phone addition tendency. The scale was scored by Likert 5 points $(1=$ very inconsistent, $5=$ very consistent $)$. The 16 items captured four dimensions, including withdrawal symptoms, prominent behavior, social comfort and mood change. In present study, the Cronbach's $\alpha$ is 0.916 and the KMO value is 0.914 .

\subsubsection{Positive Affect and Negative Affect Scale}

Positive Affect and Negative Affect Scale (PANAS) was used to measure emotion (Qiu et al., 2008). The scale consisted of 9 positive and 9 negative emotional descriptive words with a total of 18 items. The scale was scored from 1 (very inconsistent) to 5 (very consistent). The reliability of the questionnaire is good, the $\alpha$ coefficient is 0.831 and the KMO value is 0.896 .

\subsubsection{The Scale of Regulatory Emotional Self-Efficacy}

A modified version of Caprara's scale of regulatory emotional self-efficacy (SRESE, Wang et al., 2013) was used. The scale is a two-step five-factor scale with five points scoring method ( 1 = very inconsistent, $5=$ very consistent). The two levels are perceived self-efficacy in expressing positive affect (POS) and perceived self-efficacy in managing negative affect (NEG) and the five factors are perceived self-efficacy in expressing happy, perceived self-efficacy in expressing pride, perceived self-efficacy in managing irritation, perceived self-efficacy in managing despondency and perceived self-efficacy in managing guilt. In this study the Cronbach's $\alpha$ is 0.878 and the KMO value is 0.893 .

\subsection{Procedure and Data Analysis}

The research data was collected online through questionnaire stars. SPSS18.0 was used for data analysis.

\section{Results}

\subsection{Descriptive Statistics, Differences and Correlations}

Descriptive analysis showed that the mobile phone addiction tendency of college students was average $(\mathrm{M}=43.798$, $\mathrm{SD}=12.095$, median value $=44.00$ ) and the probability of cell phone addiction tendency was $40.86 \%$. The each dimension of mobile phone addition from high to low were withdrawal symptoms, social comfort, mood change and prominent behavior; and there was no significant difference in variables of gender, grade, major type, household registration type (rural and urban) and whether only child.

In this study there were 252 subjects showed positive emotional state $(\mathrm{M}=9.679, \mathrm{SD}=7.011$, median value=8.50), 77 subjects showed negative emotional state $(\mathrm{M}=5.338, \mathrm{SD}=5.736$, median value $=4.00)$.

This study suggested that self-efficacy of emotion regulation in college students was media $(\mathrm{M}=58.968, \mathrm{SD}=8.199$, median value $=57.00$ ). The self-efficacy of positive emotion regulation and negative emotion regulation are both in the middle level. The self-efficacy of expressing pride emotion is higher than that of expressing happiness emotion and the order of self-efficacy of negative emotions from high to low is management guilt self-efficacy, management depression self-efficacy and management anger self-efficacy.

Correlation analysis results showed that the mobile phone addiction tendency of college students was negatively correlated with positive emotion $(\mathrm{r}=-0.273)$, and positively correlated with negative emotion $(\mathrm{r}=0.302)$, which was consistent with the hypothesis 1 and hypothesis 2 . Positive emotion was positively correlated with regulatory emotional self-efficacy $(r=0.413)$, while negative emotion was not significantly correlated with regulatory emotional self-efficacy $(r=-0.150)$. There was no significant correlation between regulatory emotion self-efficacy and mobile phone addiction tendency $(r=0.083)$. The results of descriptive statistics and correlation matrix of variables were shown in Table 1. 
Table 1. Descriptive statistics and correlations of mobile phone addiction tendency, emotional state and regulatory emotion self-efficacy

\begin{tabular}{ccccc}
\hline Variables & 1 & 2 & 3 & 4 \\
\hline 1. Mobile phone addiction tendency & - & & & \\
2. Positive emotion & $-0.273^{* *}$ & - & & \\
3. Negative emotion & $0.302^{* *}$ & & - & \\
4. Regulatory emotion self-efficacy & 0.083 & $0.413^{* *}$ & -0.150 & - \\
$M$ & 43.798 & 9.679 & 5.338 & 58.968 \\
$S D$ & 12.095 & 7.011 & 5.735 & 8.199 \\
\hline
\end{tabular}

${ }_{p}<.05, * * p<.01, * * * p<.001$, the same below.

3.2 Mediating Effect of Regulatory Emotion Self-Efficacy on Mobile Phone Addiction Tendency and emotional State

SPSS macro program PROGRESS was used to analyze whether regulatory emotional self-efficacy played an intermediary role between mobile phone addiction tendency and emotional state. Regression analysis showed in Table 2 . The results indicated that positive emotion could significantly positively predict regulatory emotional self-efficacy ( $\beta=$ $0.483, \mathrm{p}<0.001)$ and significantly negatively predict mobile phone addiction tendency $(\beta=-0.634, p<0.001)$, and regulatory positive emotional self-efficacy could significantly positively predict mobile phone addiction tendency $(\beta=0.346, p<0.001)$. Negative emotion had no predictive effect on regulatory emotion self-efficacy $(\beta=0.211)$; but significantly positively predicted mobile phone addiction tendency $(\beta=0.831, \mathrm{p}<0.05)$; and regulatory positive emotion self-efficacy had no predictive effect on mobile phone addiction tendency $(\beta=0.355)$.

Table 2. Mediating effect of regulatory emotion self-efficacy on mobile phone addiction tendency and emotional state

\begin{tabular}{|c|c|c|c|c|c|c|}
\hline Outcome variables & $\begin{array}{l}\text { Independent } \\
\text { variables }\end{array}$ & $R$ & $R^{2}$ & $F$ & $\beta$ & $t$ \\
\hline \multirow[t]{3}{*}{$\begin{array}{l}\text { Regulatory emotion } \\
\text { self-efficacy }\end{array}$} & $\begin{array}{l}\text { Positive } \\
\text { emotion }\end{array}$ & 0.413 & 0.171 & 51.445 & 0.483 & $7.173^{* * *}$ \\
\hline & $\begin{array}{l}\text { Negative } \\
\text { emotion }\end{array}$ & 0.150 & 0.026 & 1.725 & 0.211 & 0.193 \\
\hline & $\begin{array}{l}\text { Positive } \\
\text { emotion }\end{array}$ & 0.345 & 0.119 & 16.792 & $\begin{array}{c}-0.63 \\
4\end{array}$ & $-5.626^{* * *}$ \\
\hline \multirow[t]{2}{*}{$\begin{array}{l}\text { Aobile phone addiction } \\
\text { tendency }\end{array}$} & $\begin{array}{l}\text { Negative } \\
\text { emotion }\end{array}$ & 0.361 & 0.130 & 5.547 & 0.831 & $3.028^{* *}$ \\
\hline & $\begin{array}{l}\text { Regulatory } \\
\text { emotion } \\
\text { self-efficacy }\end{array}$ & & & & $0.346(0.355)$ & $3.590^{* * *}(1.826)$ \\
\hline
\end{tabular}

The results of mediation effect analysis were shown in table 3 . The indirect effect value of positive emotion on mobile phone dependence was 0.167 , and the $95 \%$ deviation correction confidence interval did not contain 0 [0.056, 0.291]. It indicated that the mediating effect was significant. Because the direct effect of positive emotion on mobile phone dependence was significant, the self-efficacy of emotion regulation played a partial mediating role between positive emotion and mobile phone dependence. The indirect effect of negative emotion on mobile phone dependence was -0.075 , and the $95 \%$ deviation correction confidence interval contains 0 [- $0.222,0.105]$, indicating that the mediating effect was not significant. This result partially conforms to hypothesis 3 of this study. 
Table 3. Indirect effects of regulatory emotion self-efficacy on mobile phone addiction tendency and emotional state

\begin{tabular}{ccccc}
\hline & & $\beta$ & $\begin{array}{c}\text { Bootstrap } \\
\text { lower limit }\end{array}$ & $\begin{array}{c}\text { Bootstrap } \\
\text { upper limit }\end{array}$ \\
\hline Positive emotion & Direct effect & -0.634 & -0.856 & -0.412 \\
& Indirect effect & 0.167 & 0.056 & 0.291 \\
\multirow{3}{*}{ Negative emotion } & Direct effect & 0.831 & 0.284 & 1.378 \\
& Indirect effect & -0.075 & -0.222 & 0.105 \\
\hline
\end{tabular}

\section{Discussion}

\subsection{College Students' Mobile Phone Addiction Tendency Was Serious and Had no Demographics Differences}

This study found that the rate of mobile phone addiction was $40.86 \%$, significantly higher than previous $25 \%$ ( $F u, ~ L i$, $\mathrm{Xu}$ and Wei, 2016). The reason may be that more and more people rely on mobile phones. With the increasing of the daily functions of mobile phones, people use mobile phones more frequently, and waste more and more time on mobile phones. The results of demographics showed that there was no significant difference of gender, major, grade, type of residence registration, and only children in the mobile phone addiction tendency of college students. The results of this study are consistent with previous studies $((\mathrm{Fu}, \mathrm{Li}, \mathrm{Xu}$ and Wei, 2016) $)$. It showed that cell phone addiction tendency is a common problem.

\subsection{Most College Students Are in a State of Positive Emotions, and Their Regulatory Emotion Self-Efficacy Is the Average.}

This study found that $72.0 \%$ of college students were in a state of positive emotion, $6.0 \%$ were in a state of neutral emotion, and $22 \%$ were in a state of negative emotion. The rate of negative emotion in this study was lower than that of other Chinese studies. Liu et al. (2019) reported that the incidence of depression was $33.51 \%$ among 734 Chinese college students; $\mathrm{Li}$ (2018) reported that the rate of depression among 1581 Chinese college students was $48.70 \%$; Zhou \& Dou (2019) reported that the negative emotions dominated by depression and anxiety were on the rise among the 9524 Chinese college students enrolled in 2008-2017. Different measurement tools, survey methods, sample sizes, durations and so on may cause this difference.

The results showed that college students' regulatory emotion self-efficacy was average, and their ability to relieve stress and improve interpersonal relationships was stronger. In addition, the score of perceived self-efficacy in expressing positive affect was higher than that of perceived self-efficacy in managing negative affect. This showed that the ability of college students to express their positive emotions was stronger than that of managing their negative emotions.

\subsection{Emotional State Was Closely Related to Regulatory Emotion Self-Efficacy and Mobile Phone Addiction Tendency}

This study showed that college students' mobile phone addiction tendency was significantly negatively correlated with positive emotion, and positively correlated with negative emotion. This indicated that the lower the mood, or the higher the negative emotion, the more serious the mobile phone addiction tendency was, which was more consistent with the related researches. But other study also showed that a high degree of depression was related to the less use of mobile phones because severe depression tended to social avoidance (Elhai et al., 2018).

Positive emotion had a significant positive correlation with self-efficacy of emotion regulation, which indicated that in the state of positive emotion, self-efficacy of emotion regulation will increase. Social cognitive theory of emotional regulation thought believing that you can change your current mood is an important factor in the successful regulation of negative emotions (Tamir \& Mauss., 2011). That is to say, individuals with strong regulatory emotion self-efficacy can weaken the reaction of negative emotions and enhance the expression of positive emotions, which shows the influence of regulatory emotion self-efficacy on emotional state.

\subsection{The Mediating Role of Regulatory Emotion Self-Efficacy Between Mobile Addiction Tendency and Emotional State}

This study suggested that in a state of positive emotion regulatory emotion self-efficacy has direct effect on mobile phone addiction and it also played a partially mediating role in the relationship between emotional state and mobile phone addiction tendency. This shows that the stronger the positive state of emotion is, the stronger the regulatory emotion self-efficacy is. However, in the negative emotional state, regulatory emotion self-efficacy did not show mediating effect.

\section{Conclusion}

Survey was used to explore the status quo of college students' emotional state, regulatory emotion self-efficacy and mobile phone addiction tendency and their relationships. The result indicated that: (1) 40.86\% of college students had 
the tendency of cell phone addiction, which was serious; $72.0 \%$ of college students were in a positive emotional state, $22 \%$ were in a negative emotional state; college students' regulatory emotion self-efficacy was in the middle level; (2) there was no gender, grade, major type, household registration type (rural and urban) and whether only child difference in mobile phone addiction tendency; (3) the positive emotions of college students were negatively correlated with the tendency of mobile phone addiction, while the negative emotions were positively correlated with the tendency of mobile phone addiction, and the positive emotion was positively correlated with regulatory emotional self-efficacy; (4) The regulatory emotion self-efficacy had a partial mediating effect between the positive emotions and mobile phone addiction tendency and had no mediating effect between the negative emotions and mobile phone addiction tendency.

This work had certain innovation. The results laid a good foundation for further exploring the internal psychological mechanism of mobile phone addiction. Therefore, helping college students to deal with their emotional state will be a more effective way to control the tendency of mobile phone addiction. Nevertheless, this study still has limitation. The sample size is not large enough, and the distribution of samples in gender, grade and other variables is not uniform enough, which may affect the analysis results. Therefore, future research needs to focus on a larger sample size. In addition, the classification of emotions can only be divided into positive and negative categories, without exploring the specific emotions.

\section{Acknowledgements}

This work was supported by The National Social Science Fund of China [grant number 16BSH094].

\section{References}

Bandura, A. (1986). Social foundations of thought and action: asocial cognitive theory. Englewood Cliffs, New Jersey: Prentice-Hall.

Bandura, A., Caprara, G. V., Barbaranelli, C., Gerbino, M., \& Pastorelli, C. (2003). Role of affective self-regulatory efficacy on diverse spheres of psychosocial functioning. Child Development, 74(3), 769-782. https://doi.org/10.1111/1467-8624.00567

Bianchi, A., \& Phillips, J. G. (2005). Psychological predictors of problem mobile phone use. Cyberpsychol Behav, 8(1), 39-51. https://doi.org/10.1089/cpb.2005.8.39

Caprara. G. V., S., E., Barbarnelli, C., Pastorelli, C., Regalia, C., \& Bandura, A. (1999). Perceived emotional and interpersonal self-efficacy and good social functioning. GiornaleItaliano di Psicologia, 26, 769-789.

Chai, J. (2017). The behavior intention and impact factors of mobile phone involvement in university student. http://www.jlu.edu.cn

Coyne, S. M., Stockdale, L., \& Summers, K. (2019). Problematic cell phone use, depression, anxiety, and self-regulation: Evidence from a three year longitudinal study from adolescence to emerging adulthood. Computers in Human Behavior, 96, 78-84. https://doi.org/10.1016/j.chb.2019.02.014

Elhai, J. D., Tiamiyu, M. F., Weeks, J. W., Levine, J. C., Picard, K. J., \& Hall, B. J. (2018). Depression and emotion regulation predict objective smartphone use measured over one week. Personality and Individual Differences, 133, 21-28. https://doi.org/10.1016/j.paid.2017.04.051

Elhai, J. D., Yang, H., Fang, J., Bai, X., \& Hall, B. J. (2019). Depression and anxiety symptoms are related to problematic smartphone use severity in Chinese young adults: Fear of missing out as a mediator. Addict Behav, 105962. https://doi.org/10.1016/j.addbeh.2019.04.020

Fu, G. F., Li, X. Y., Xu, Z. K., \& Wei, Xi. J. (2016). A survey of mobile phone dependence of college students. Journal of higher education, 2016(01), 65-68.

Ikeda, K., \& Nakamura, K. (2014). Association between mobile phone use and depressed mood in Japanese adolescents: a cross-sectional study. Environmental Health and Preventive Medicine, 19(3), 187-193. https://doi.org/10.1007/s12199-013-0373-3

Lepp, A., Barkley, J. E., \& Karpinski, A. C. (2015). The Relationship Between Cell Phone Use and Academic Performance in a Sample of U.S. College Students. SAGE Open, 5(1). https://doi.org/10.1177/2158244015573169

Li, Y. X., Xie, J. Z., Gao, X., Li, X. Z., Zhang, Q., Cao, L. L., \& Xuan, Y. (2018). The research of the depression condition and its influence factors among college students in Qinghai University. Henan Journal of Preventive Medicine, 29(01), 8-12.

Liu, M., Ni, F. D., \& Ma, S. F. (2019). Investigation on Depression of College Statistical Bulletin of the Communications Operations Industry in http://www.miit.gov.cn/n1146285/n1146352/n3054355/n3057511/n3057518/c6618525/content.html 
Park., W. K. (2005). Mobile phone addiction [C]/ Mobile Communications, Renegotiation of the Social Sphere. London: Springer, 253-272. https://doi.org/10.1007/1-84628-248-9_17

Qiu Lin, Z. X., \& Wang Yanfei. (2008). Revision of the Positive Affective Negative Affective Scale (PANAS). Chinese Journal of Applied Psychology, 14(03), 249-254+268.

Students in a University of Gansu Province. South China Journal of Preventive Medicine, 45(01), 69-72.

Tamir M., M. I. B. (2011). Social Cognitive Factors in Emotion Regulation: Implications for Well-Being. In: Nyklíček I., Vingerhoets A., Zeelenberg M. (eds) Emotion Regulation and Well-Being. Springer, New York, NY. https://doi.org/10.1007/978-1-4419-6953-8_3

Tang, D. L., Yu, G. L., \& Wen, S. F. (2010). Regulatory Emotion Self-efficacy: A New Research Topic. Advances in Psychological Science, 18(04), 598-604.

Wang, Y. J., \& Liu, Y. (2013). Revision of Regulatory Emotional Self-Efficacy Scale. Journal of Guangzhou University (Social Science Edition), 12(01), 45-50.

Wen, S. F., \& Yu, G. L. (2009). Applied Research on Regulatory Emotion Self-Efficacy. Psychological Science, 32(3), 666-668.

Xiong, J., Chen, W., You, Z. Q., \& Zhai, Z. Y. (2012). Development of the Mobile Phone Addiction Tendency Scale for College Students. Chinese Mental Health Journal, 26(3), 222-225.

Zhang, B., Jiang, H. B., \& Cui, R. H. (2015). The relationship between loneliness, negative emotion and cell phone addiction in College Students. Journal of Jimei University (Education Science Edition), 16(1).

Zhang, L. R., Zhang, C. C., Dai, Y., \& Zhu, X. (2018). A review on mobile phone addiction among college students. Chinese Journal of Health Education, 34(01), 59-61+89.

Zhou, S. X. (2019). New Trends of College Students' Emotional Characteristics under Social Changes and Countermeasures: A Case Study of a University in Beijing. Journal of Chinese Youth Social Science, 38(04), 59-66.

\section{Copyrights}

Copyright for this article is retained by the author(s), with first publication rights granted to the journal.

This is an open-access article distributed under the terms and conditions of the Creative Commons Attribution license which permits unrestricted use, distribution, and reproduction in any medium, provided the original work is properly cited. 